\title{
Addition of mannitol to hyaluronic acid enhances the inhibition of matrix metalloprotease expression in IL-1 $\beta$ stimulated human C-20/A4 chondrocytes
}

\author{
L Grange $^{1,2}$, J. Kessler ${ }^{3}$ and T Conrozier ${ }^{3 *}$ \\ ${ }^{1}$ Service de Rhumatologie, CHU Grenoble-Alpes, France \\ ${ }^{2}$ GREPI AGIM FRE3405, CNRS-UJF-EPHE, France
}

${ }^{3}$ Service de Rhumatologie, Hopital Nord Franche-Comté, France

*Corresponding author: Thierry Conrozier, Department of Rheumatology, Hopital Nord Franche-Comté, 1àà route de Moval, CS 10499

Trévenans, 90000, Belfort, France

Keywords: Chondrocyte; Mannitol; Hyaluronic Acid; Metalloprotease; In Vitro Study

\section{Introduction}

Osteoarthritis $(\mathrm{OA})$ of the knee and hip is ranked as the $11^{\text {th }}$ leading raison of disability and its prevalence keeps going up over the last past decades [1]. In the United States, over 9 million people suffer from symptomatic knee OA [2]. One third of adults over the age of 60 have radiographic evidence of this condition and $12.1 \%$ experience pain and/or disability [3]. Despite the high prevalence of knee $\mathrm{OA}$ and the high level of disability related to the disease progression, only few patients undergo joint replacement with a mean time from symptoms onset to Total Knee Replacement (TKR) of about 19 years $[3,4]$. Such a long evolution explains the huge need of new non-surgical therapies, which can alleviate pain and improve the quality of life with a better effectiveness than the current therapeutic modalities. The therapeuticapproach of knee OAis based on the combination of non-pharmacological and pharmacological modalities, widely described in multiple guidelines [5-7]. Among the pharmacological modalities, Intra-Articular (IA) injections of hyaluronic acid (HA), also named Viscosupplementation (VS), have been ranked as the most effective treatment for alleviating knee OA pain [8], despite some controversies remain regarding the interpretation of the meta-analyses [9-12]. Hyaluronic acid is a non sulfated High Molecular Weight (HMW) glycosaminoglycan naturally present in ECM and synovial fluid that plays a major role in cartilage lubrication $[13,14]$. The HA lubricating properties explain that $\mathrm{HA}$ injections for treatment of $\mathrm{OA}$ are classified by the majority of health agencies worldwide, as a medical device but not as a drug. However, it has been now well evidenced that IA HA has also multiple pharmacological effects on molecular signaling pathways and contributes to the joint homeostasis. The multiple mechanisms by which HA may act as an anti-inflammatory and a structure-modifying drug for $\mathrm{OA}$ have been developed in a recent review [15].

Inflammation plays a central role in the development and progression of $\mathrm{OA}$. Multiple pro-inflammatory cytokines are involved in the pathogenesis of the disease, especially interleukin (IL)-1 $\beta$, IL-6, and tumor necrosis factor (TNF)- $\alpha$, which play key roles in the extra cellular matrix (ECM) breakdown. IL-1 $\beta$ and TNF- $\alpha$, produced by chondrocytes, synoviocytes, mononuclear cells and even osteoblasts, stimulate matrix metalloproteinases (MMPs) and a disintegrin and metalloproteinase with thrombospondin motifs (ADAMTS) [16] synthesis and activity. Furthermore IL-1 $\beta$ stimulates Nitric Oxide (NO) synthesis and oxygen free radicals production that increases the damaging effects on cartilage [17]. The ECM degradation and particularly that of its two major components, aggrecan and type 2 collagen, is mainly due to MMP-1, $-3,-9$, and -13 , and ADAMTs 4 and 5 . Hyaluronidase and Reactive 
Oxygen Species (ROS) degrade HA macromolecules, leading to increased levels of HA fragments of low molecular weight with pro-inflammatory properties that worsen again the inflammatory cascade [15]. The important role of oxidative stress resulting from chronic overproduction of ROS is often underestimated. Oxygen free radicals damage DNA, increase cell apoptosis, contribute to degradation of the ECM and inhibit proteoglycans synthesis [18]. The NADPH oxidase enzymes constitute a large enzyme family which function is dedicated to the production of reactive oxygen species (ROS). One of our recent work showed that the NADPPH oxidase 4 (Nox4) is the major source of ROS production in human chondrocytes and in the C-20/A4 human chondrocyte cell line $[19,20]$ in which Nox4-derived ROS regulate collagenase 1 (MMP-1) expression and chondrocyte death consecutive to IL-1 $\beta$ stimulation. Interestingly a number of hydroxyl rich polysaccharides, such as HA, have also antioxidant properties and are themselves inhibitors of oxygen free radical $-02 *$, underlining the importance of $\mathrm{HA}$ concentration [21]. However HA is also very sensitive to oxidative stress [22]. HA macromolecules, containing many OH groups, react with ROS, resulting in the rupture of the macromolecular chains and accelerated degradation of the highly viscous solution. The rapid depolymerisation of HA is a major reason for the short intraarticular half-life of viscosupplements made of non-cross-linked $\mathrm{HA}$, cross-linking being another way to protect HA from degradation by ROS [21]. Several studies have shown that the addition of a polyol with ROS scavenging properties, such as mannitol or sorbitol, protect HA from ROS degradation [21-24]. Safety and efficacy of a mannitol-modified HA viscosupplement, HANOX-M, has been assessed in a large scale multicenter, prospective doubleblind controlled trial [25] in patients with knee OA. The study demonstrated that HANOX-M was as safe and effective than a high molecular weight $\mathrm{HA}$ viscosupplément not containing mannitol, but with a more rapid onset of action than that of the latter [26]. Due to the antioxidant effect of mannitol we hypothesized that, besides its better resistance to degradation, the mannitol-modified HA might have a more pronounced effect than HA alone on inflammation and MMPs related ECM degradation. The aim of the study was to compare in vitro the effect of the addition of mannitol to HA versus HA alone on the level of ROS production and the NOX-4 and MMPs expression upon IL-1 $\beta$ stimulation.

\section{Materiel and Methods}

\section{Material}

Human C-20/A4 chondrocyte cell line immortalized by SV40 is a generous gift from Dr M.B. Goldring (Harvard Institute of Medicine, Boston, MA, USA). HEK293 Nox4 T-Rex ${ }^{\text {TM }}$ cells were kindly provided by PATIM laboratory, Pr KH. Krause (Geneva University, Switzerland). Dulbecco's modified Eagle's medium (DMEM), fetal bovine serum (FBS) and geneticin (G418) were purchased from life technologies (Saint Aubin, France); blasticidin was from Funakoshi
Co (Tokyo, Japan); complete mini EDTA-free protease inhibitor EASYpack, $\mathrm{Na}_{4} \mathrm{P}_{2} \mathrm{O}_{7}, \mathrm{Na}_{3} \mathrm{VO}_{4}, \mathrm{PMSF}$, luminol, Horseradish Peroxidase (HRPO), Triton X-100, were purchased from SIGMA (Saint Quentin Fallavier, France) ; leupeptin, pepstatin, trypsin inhibitor, TLCK and human interleukin-1 $\beta$ were purchased from Roche (Meylan, France) ; Diisopropylfluoro-phosphate (DFP) was purchased from Acros Organics (Halluin, France); Pro-MMP1 monoclonal antibody was obtained from R\&D Systems (Lille, France); rabbit polyclonal antibodies directed against ADAMTS5 (RP1-ADAMTS5) were respectively from Biovalley (Illkirch, France) and Abcam (Paris, France).

\section{Hyaluronic Acid Products}

The viscosupplement to be studied was HAnox-M (HAppyVisc $®$, LABRHA SAS, Lyon, France), a solution made of an intermediate MW (1-1.5MDa) HA of non-animal origin, in a concentration of 1.55 $\mathrm{mg} / \mathrm{ml}$, combined with mannitol, concentrated at $35 \mathrm{mg} / \mathrm{ml}$. The comparator was HA1\% (Go On $\AA$, Meda Pharma, Solna,Sweden), solution of an intermediate MW (1-1.5 MDa) of non-animal origin, in a $10 \mathrm{mg} / \mathrm{ml}$ concentration.

\section{Cell culture}

C-20/A4 chondrocytes and HEK293 T-Rex ${ }^{\mathrm{TM}}$ were cultured in DMEM containing $4.5 \mathrm{~g} / \mathrm{L}$ glucose and $0.11 \mathrm{~g} / \mathrm{L}$ sodium pyruvate, supplemented with $10 \%(\mathrm{v} / \mathrm{v})$ fetal bovine serum, 100 units $/ \mathrm{ml}$ penicillin, $100 \mathrm{mg} / \mathrm{ml}$ streptomycin and $2 \mathrm{mM}$ glutamine at $37^{\circ} \mathrm{C}$ in atmosphere containing $5 \% \mathrm{CO}_{2}$. Selecting antibiotics, blasticidin $(5 \mu \mathrm{g} / \mathrm{ml})$ and geneticine $(400 \mu \mathrm{g} / \mathrm{ml})$ were used for HEK293 Nox4 T-Rex ${ }^{\mathrm{TM}}$ cells. Expression of Nox4 by HEK293 Nox4 T-Rex ${ }^{\mathrm{TM}}$ cells were induced by the addition of $1 \mu \mathrm{g} / \mathrm{ml}$ tetracycline in the culture media [26]. All experiments were performed within cell passages 3 to 10 at $60-90 \%$ confluence. Cells were incubated in presence of HANOX-M or HA $1 \%$ e with a final concentration of $1.25 \mathrm{mg} / \mathrm{ml}$ hyaluronic acid. Cells were stimulated or not with $2 \mathrm{ng} / \mathrm{ml}$ IL-1 $\beta$ during $48 \mathrm{~h}$ for RT-PCR or $24 \mathrm{~h}$ for ROS production evaluation.

\section{Evaluation of ROS Production}

Expression of Nox 4 by HEK293 Nox4 T-Rex ${ }^{\mathrm{TM}}$ cells were induced by the addition of $1 \mu \mathrm{g} / \mathrm{ml}$ tetracycline in the culture media for 24 hours. Cells were then detached with $0.25 \%$ (w/v) trypsin, washed twice with PBS and collected after 8 min centrifugation at $400 \mathrm{~g}$ at room temperature. The viability of the suspended cells was over $95 \%$, as determined by the trypan blue exclusion method. In a 96well plate, $5 \times 10^{5}$ living cells resuspended in $20 \mu \mathrm{l}$ PBS were added per well. Before the start of the assay, $100 \mu$ of a PBS solution containing $5 \mu \mathrm{M}$ Amplex Red and 10 mUnits/ml horseradish peroxidase was added in each well. Results are expressed as the sum of Relative Fluorescent Units (RFU) recorded every two minutes during $60 \mathrm{~min}$ on a fluostar omega spectrofluorimeter (BMG labtech). 


\section{RNA Extraction and RT-q-PCR}

Total RNA extraction was performed with NucleoSpin $\left({ }^{\circledR}\right.$ RNA (Macherey Nagel, Hoerd, France) following recommendations. cDNA was reverse transcribed from $1 \mu \mathrm{g}$ of total RNA with the SuperScriptIII First-Strand Synthesis (Life Technologies, Carlsbad, CA). As recommended an RNase $\mathrm{H}$ treatment was added. Real time RT-qPCR was conducted using the QuantiTect SYBR Green RT-PCR kit (Qiagen) and a Stratagene Mx3005P (La Jolla, CA). Briefly, the transcript expression levels of human IL-1 $\beta$, CYBA, NOX4, HMOX1, MMP-1, MMP-13, ADAMTS4 and housekeeping genes GAPDH, RPL27 and RPL32 were determined using specific primers chosen to include intron spanning. Gene expression was quantified using the comparative threshold cycle (Ct) method. The amount of target gene, normalized to three endogenous reference genes (RPL27, RPL32 and GAPDH) was expressed relative to the control cells. The specificity of the PCR products was confirmed by gel electrophoresis migration and a by melting curve analysis.

\section{Statistical Analysis}

Data are presented as means +/- SD, significance levels were assessed using Student's paired t test. A p-value of 0.05 or less between groups was considered to indicate a statistically significant difference.
All quantitative data were generated using biological replicates in triplicate unless stated otherwise and are expressed as the mean plus 95\% confidence interval. Data normality was tested by a Shapiro-Wilk test. For each experiment, p-values were determined using a paired Student test. All analyses were conducted using StatView (SAS institute, US).

\section{Results}

\section{Differential Impact Of HANOX-M And HA 1\% On NADPH Oxidase Activity In HEK293 T-REX ${ }^{\mathrm{TM}}$ Cells}

When HANOX-M or HA 1\%, at a final concentration of $1.25 \mathrm{mg} /$ $\mathrm{ml}$ of HA, were added to the cells right before the ROS production measurement, or 24 hours before ROS measurement, no significant impact on Nox4 activity was noticeable, showing that the two viscosupplement compounds do not exert direct antioxidant properties.(Data not shown)

\section{Effect Of HANOX-M And HA 1\% On Nox4 Expression In Human Chondrocyte Cell Line C20/A4}

As expected, IL-1 $\beta$-stimulated C-20/A4 chondrocyte cell line increase Nox4 expression, determined by quantitative PCR. Despite a non-statistically significant difference, HANOX-M tended to better inhibit Nox4 expression than HA 1\% (Figure 1).

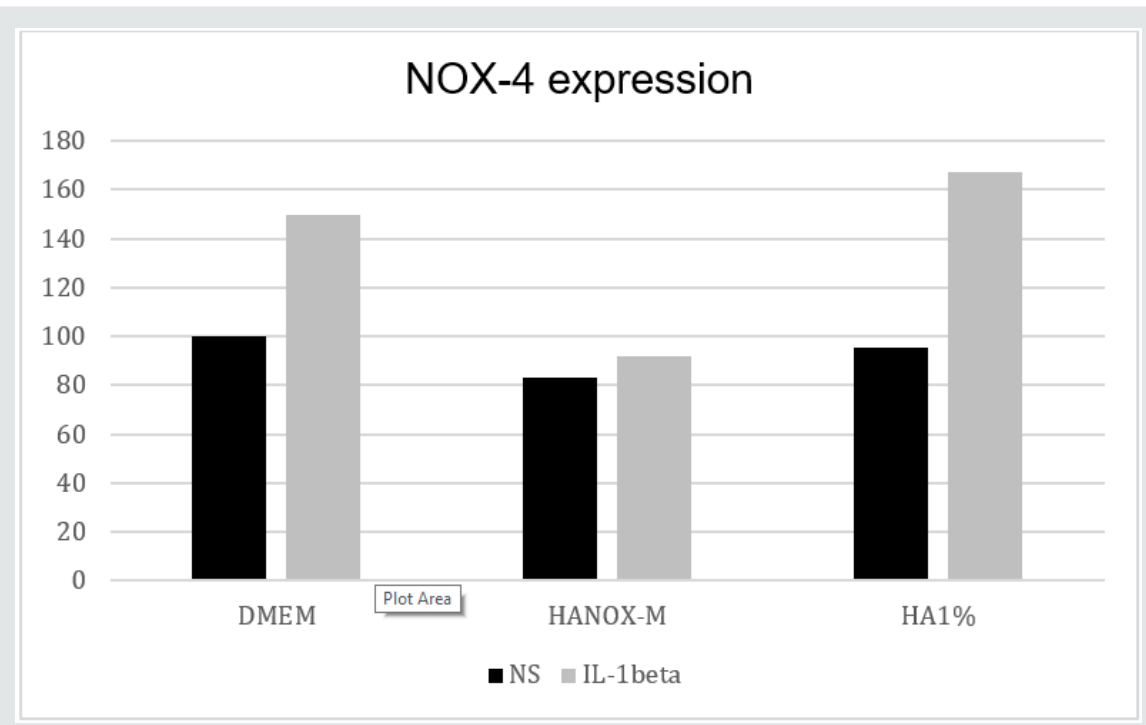

Figure 1: NOX-4 expression in non-stimulated (NS) and IL-1ß-stimulated HEK 293 Nox4 ${ }^{\mathrm{TM}}$ cells, after incubation with HANOX-M or HA1\%.

\section{Effect of HANOX-M and HA 1\% on MMPs expression in} human chondrocyte cell line $\mathrm{C} 20 / \mathrm{A} 4$

Figures 2, 3, 4 Quantitative RT-PCR showed that treatment with HANOX-M inhibits the effect of stimulation by IL1 $\beta$ on the increase in expression of MMP13 and ADAMTs4. Treatment with HA1\%, on the other hand, cannot prevent the increase in transcripts encoding the two metalloproteases MMP13 and ADAMTs4.

The effect of stimulation by IL1 $\beta$ on the increase in expression of MMP1 was completely inhibited by the two treatments, HANOX-M and HA1\% (Figure 4). 


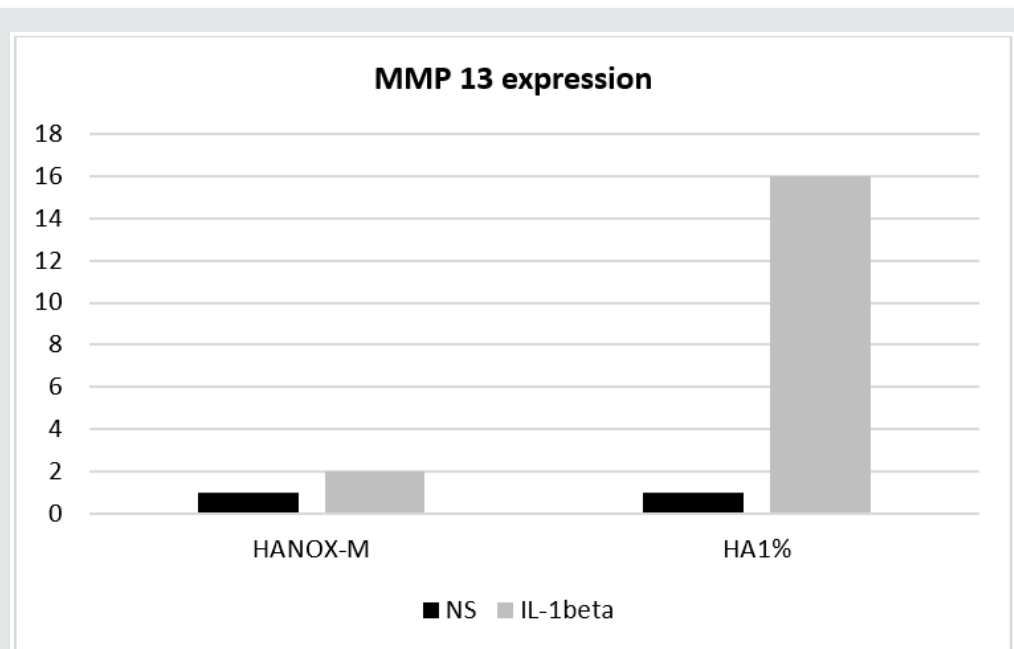

Figure 2: Collagenase MMP13 expression in non-stimulated (NS) and IL-1 $\beta$-stimulated C20/ A4 chondrocytes, after incubation with HANOX-M or HA1\%.

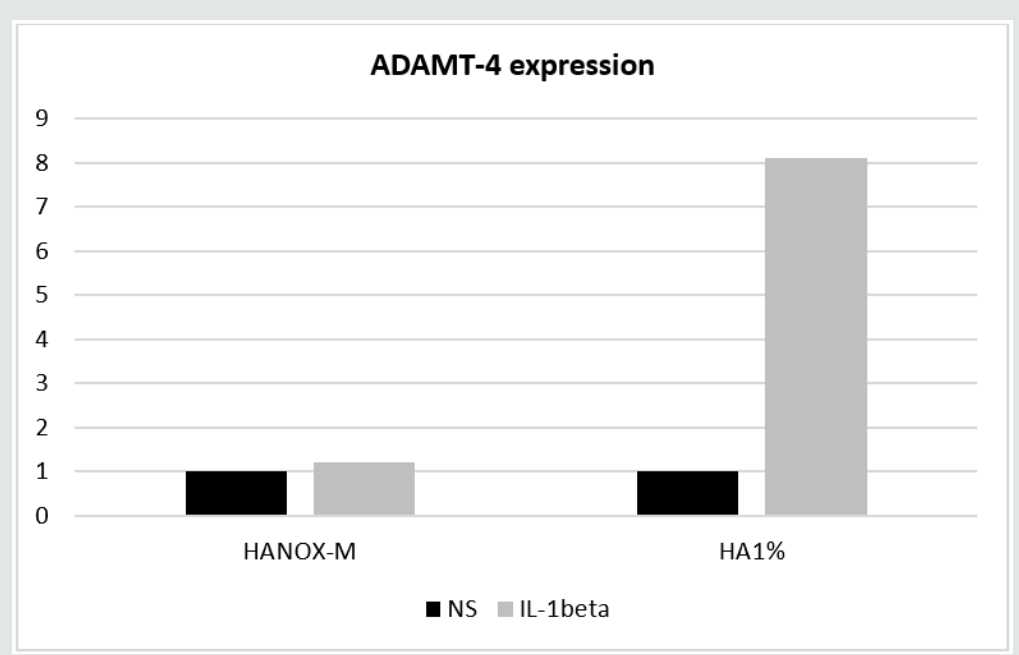

Figure 3: Aggrecanase ADAMTS-4 expression in non-stimulated (NS) and IL-1 $\beta$-stimulated C20/A4 chondrocytes, after incubation with HANOX-M or HA1\%.

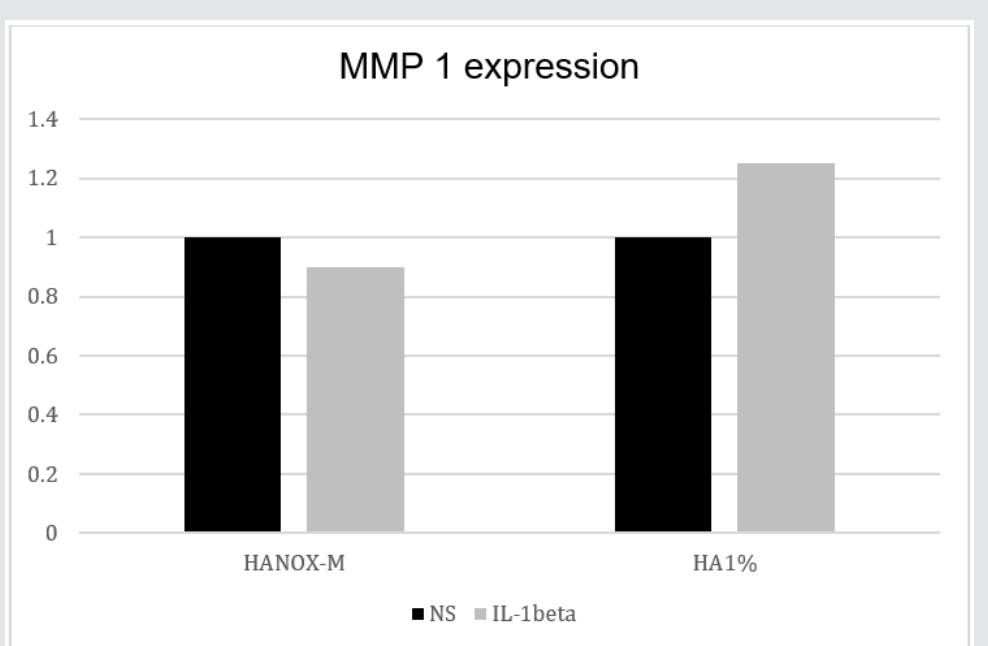

Figure 4: Collagenase MMP1 expression in non-stimulated (NS) and IL-1 $\beta$-stimulated C20/A4 chondrocytes, after incubation with HANOX-M or HA1\%. 


\section{Discussion}

It has become increasingly apparent that $\mathrm{HA}$ has the capacity not only to alleviate pain but also to protect and restore the chondral matrix via multiple molecular pathways [15]. Therefore it is legitimate to consider HA not only as a pain-killer but as a background therapy for $\mathrm{OA}$ with structure-modifying properties. The development of novel HA viscosupplements with chondroprotective effects, especially for treating early $\mathrm{OA}$, is a exciting challenge. Henrotin et al. (27) reported the results of a randomized double-blind placebo controlled trial showing that a single IA injection of HANOX-M-XL, a crosslinked HA combined with mannitol, can reduce the serum levels of Coll2-1, a marker specific of type II collagen degradation, in patients with knee OA. This finding suggests that HANOX-M-XL may have a beneficial effect on cartilage degradation.The present study compared the effects of HANOX-M, a non-crosslinked $2^{\text {nd }}$ generation viscosupplement [28], containing $35 \mathrm{mg} / \mathrm{ml}$ of mannitol, to a regular $\mathrm{HA} 1 \%$ viscosupplement. It suggested that HANOX-M might have a positive therapeutic effect on the expression of the transcripts coding for the metalloproteinases MMP1, MMP13 and ADAMTs4, while HA1\% exhibited a positive effect only on the expression of MMP1. HANOX-M also tended to better inhibit Nox4 expression than HA $1 \%$, despite a non-statistically significant between-treatment difference. The mechanisms by which mannitol exerts its beneficial effect on OA are not fully understood. It has been published [29] that a combination of HA and sorbitol, a mannitol isomer, prevented IL$1 \beta$-induced oxidative stress, p47-NADPH oxidase phosphorylation, 4-hydroxynonenal (HNE) production and HNE-metabolizing glutathione-S-transferase A4-4 expression. Furthermore, HA + sorbitol inhibited IL-1 $\beta$-induced MMP-13, nitric oxide (NO) and prostaglandin E2 release and NO synthase expression, by acting on the nuclear factor-kappa B (NFKB) pathway. Obviously, our data do not allow us to extrapolate these in vitro results to claim a chondroprotective effect of HANOX-M, in vivo.

\section{Conclusion}

This in vitro study suggests that combining high concentration of mannitol with HA may have a positive therapeutic effect on the expression of the transcripts coding for the metalloproteases MMP1, MMP13 and ADAMTs4. Treatment with HA alone has a potentially therapeutic effect only on the expression of MMP1. HANOX-M might be useful as a first line treatment to treat chondropathy and early $\mathrm{OA}$, thanks to its potential structure-modifying effect.

\section{References}

1. Cross M, Smith E, Hoy D, Sandra Nolte, Ilana Ackerman, et al. (2014) The global burden of hip and knee osteoarthritis: estimates from the global burden of disease 2010 study. Ann Rheum Dis 73(7): 1323-1330.

2. Lawrence RC, Felson DT, Helmick CG, Arnoldet LM, Choi H, al. Estimates of the prevalence of arthritis and other rheumatic conditions in the United States. Part II. Arthritis Rheum 58(1): 26-35.
3. Dillon CF, Rasch EK, Gu Q Hirsch R (1994) Prevalence of knee osteoarthritis in the United States: arthritis data from the Third National Health and Nutrition Examination Survey. J Rheumatol 33(11): 22712279.

4. Losina E, Paltiel AD, Weinstein AM, Yelin E, Hunter DJ, et al. (2015) Lifetime medical costs of knee osteoarthritis management in the United States: Impact of extending indications for total knee arthroplasty. Arthritis Care Res (Hoboken) 67(2): 203-215.

5. Jordan KM, Arden NK, Doherty M, Bannwarth B, Bijlsma JW, Dieppe P, et al (2003) EULAR Recommendations 2003: an evidence based approach to the management of knee osteoarthritis: Report of a Task Force of the Standing Committee for International Clinical Studies Including Therapeutic Trials (ESCISIT). Ann Rheum Dis 62(12): 1145-1155.

6. Hochberg MC, Altman RD, April KT, Benkhalti M, Guyatt G, McGowan J, et al (2012) American College of Rheumatology 2012 recommendations for the use of nonpharmacologic and pharmacologic therapies in osteoarthritis of the hand, hip, and knee. Arthritis Care Res; 64(4): 465474.

7. McAlindon TE, Bannuru RR, Sullivan MC, Arden NK, Berenbaum F, et al. (2014) OARSI guidelines for the non-surgical management of knee osteoarthritis. Osteoarthritis Cartilage 22: 363-388.

8. Bruyère 0 , Cooper $C$, Pelletier JP, Branco J, Brandi ML, et al. (2014) An algorithm recommendation for the management of knee osteoarthritis in Europe and internationally: A report from a task force of the European Society for Clinical and Economic Aspects of Osteoporosis and Osteoarthritis (ESCEO). Semin. Arthritis Rheum 44(3): 253-263.

9. Rutjes AW, Jüni P, da Costa BR, Trelle S, Nüesch E, et al. (2012) Viscosupplementation for osteoarthritis of the knee: a systematic review and meta-analysis. Ann Intern Med 157(3): 180-191.

10. Campbell J, Bellamy N, Gee T (2007) Differences between systematic reviews/meta-analyses of hyaluronic acid/hyaluronan/hylan in osteoarthritis of the knee. Osteoarthritis Cartilage; 15(12): 1424-1436.

11. Johansen M, Bahrt H, Altman RD, Bartels EM, Juhl CB, Bliddal H, et al. (2016) Exploring reasons for the observed inconsistent trial reports on intra-articular injections with hyaluronic acid in the treatment of osteoarthritis: Meta-regression analyses of randomized trials. Semin Arthritis Rheum 46(1):34-48.

12. Xing D, Wang B, Liu Q Ke Y, Xu Y, et al. (2016) Intra-articular Hyaluronic Acid in Treating Knee Osteoarthritis: a PRISMA-Compliant Systematic Review of Overlapping Meta-analysis. Sci Rep 6: 32790.

13. Greene GW, Banquy X, Lee DW, Lowrey DD, Yu J, et al. (2011) Adaptive mechanically controlled lubrication mechanism found in articular joints. Proc Natl Acad Sci 108(13): 5255-5259.

14. Swann DA, Radin EL, Nazimiec M, Weisser PA, Curran N, et al. (1974) Role of hyaluronic acid in joint lubrication. Ann Rheum Dis 33(4): 318326.

15. Nicholls MA, Fierlinger A, Niazi F, Bhandari M (2017) The DiseaseModifying Effects of Hyaluronan in the Osteoarthritic Disease State. Clin Med Insights Arthritis Musculoskelet Disord 10: 1179544117723611.

16. Daheshia M, Yao JQ (2008) The interleukin 1beta pathway in the pathogenesis of osteoarthritis. J Rheumatol 35(12): 2306-2312.

17. Kapoor M, Martel-Pelletier J, Lajeunesse D, Pelletier JP, Fahmi H (2011) Role of proinflammatory cytokines in the pathophysiology of osteoarthritis. Nat Rev Rheumatol 7(1): 33-42.

18. Henrotin Y, Kurz B, Aigner T (2005) Oxygen and reactive oxygen species in cartilage degradation: friends or foes? Osteoarthritis Cartilage 13(8): 643-654.

19. Grange L, Nguyen MV, Lardy B, Derouazi M, Campion Y, et al. (2006) $\mathrm{NAD}(\mathrm{P}) \mathrm{H}$ oxidase activity of Nox4 in chondrocytes is both inducible

Citation: L Grange, J. Kessler, T Conrozier. Addition of mannitol to hyaluronic acid enhances the inhibition of matrix metalloprotease expression in IL-1ß stimulated human C-20/A4 chondrocytes. Orthop \& Spo Med Op Acc J 4(2)- 2020. OSMOAJ.MS.ID.000181. DOI: 10.32474/OSMOAJ.2020.04.000181. 
and involved in collagenase expression. Antioxid Redox Signal 8(9-10): 1485-1496.

20. Rousset F, Nguyen MV, Grange L, Morel F, Lardy B (2013) Heme oxygenase-1 regulates matrix metalloproteinase MMP-1 secretion and chondrocyte cell death via Nox4 NADPH oxidase activity in chondrocytes. PLoS One 8(6): e66478.

21. Rinaudo M, Lardy B, Grange L, Conrozier T (2014) Effect of mannitol on hyaluronic acid stability in two in vitro models of oxidative stress. Polymers 6(7): 1948-1957.

22. Stern R, Kogan G, Jedrzejas MJ, Soltés L (2007) The many ways to cleave hyaluronan. Biotechnol Adv 25[6]: 537-557.

23. Mendoza G, Alvarez AI, Pulido MM, Molina AJ, Merino G, et al. (2007) Inhibitory effects of different antioxidants on hyaluronan depolymerization. Carbohydr Res 342: 96-102.

24. Conrozier T, Mathieu P, Rinaudo M (2014) Mannitol Preserves the Viscoelastic Properties of Hyaluronic Acid in an In Vitro Model of Oxidative Stress. Rheumatol Ther 1(1): 45-54.

25. Conrozier T, Eymard F, Afif N, Balblanc JC, Legré-Boyer V, et al. (2016) Safety and efficacy of intra-articular injections of a combination of hyaluronic acid and mannitol [HAnOX-M] in patients with symptomatic knee osteoarthritis: Results of a double-blind, controlled, multicenter randomized trial. Knee 23[5]: 842-848.

26. Eymard F, Bossert M, Lecurieux R, Maillet B, Chevalier X, et al. (2016) Addition of Mannitol to Hyaluronic Acid may Shorten Viscosupplementation Onset of Action in Patients with Knee Osteoarthritis: Post-Hoc Analysis of A Double-blind, Controlled Trial. J Clin Exp Orthop 2: 21.

27. Henrotin Y, Berenbaum F, Chevalier X, Marty M, Richette P, et al. (2017) Reduction of the Serum Levels of a Specific Biomarker of Cartilage Degradation (Coll2-1) by Hyaluronic Acid (KARTILAGE® CROSS) Compared to Placebo in Painful Knee Osteoarthritis Patients: the EPIKART Study, a Pilot Prospective Comparative Randomized Double Blind Trial. BMC Musculoskelet Disord 18(1): 222.

28. Conrozier T (2018) Is the Addition of a Polyol to Hyaluronic Acid a Significant Advance in the Treatment of Osteoarthritis? Curr Rheumatol Rev 14(3): 226-230.

29. Mongkhon JM, Thach M, Shi Q Fernandes JC, Fahmi H, et al. (2014) Sorbitol-modified hyaluronic acid reduces oxidative stress, apoptosis and mediators of inflammation and catabolism in human osteoarthritic chondrocytes. Inflamm Res 63(8): 691-701.

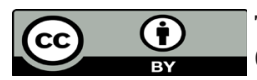

This work is licensed under Creative Commons Attribution 4.0 License

To Submit Your Article Click Here:

Submit Article

DOI: $10.32474 /$ OSMOAJ.2020.04.000181

$\begin{gathered}\text { Orthopedics and Sports Medicine } \\ \text { Open Access Journal }\end{gathered}$
Assets of Publishing with us
- Global archiving of articles
- Immediate, unrestricted online access
- Rigorous Peer Review Process
- Authors Retain Copyrights
Orthopedics And Sports Medicine:
Open Access Journal

